\title{
EW and QCD corrections at NLO with RECOLA
}

\author{
Ansgar Denner \\ Julius-Maximilians-Universität Würzburg, Germany \\ E-mail: denner@physik.uni-wuerzburg.de
}

\section{Lars Hofer}

ECM and ICCUB, Universitat de Barcelona (UB), Spain

E-mail: hofer@ecm.ub.edu

\section{Andreas Scharf}

Julius-Maximilians-Universität Würzburg, Germany

E-mail: ascharf@physik. uni-wuerzburg.de

\section{Sandro Uccirati*}

Torino University and INFN, Italy

E-mail: ucciratieto.infn.it

We present the features of the code RECOLA for the computation of NLO amplitudes for elementary processes in the full Standard Model. The code is based on recursive relations which improve the efficiency of the computation of the matrix elements, avoiding re-computation of subamplitudes. RECOLA has been used to calculate the electroweak corrections to the production of a $\mathrm{Z}$ boson in association with two jets at the LHC, including the leptonic decay of the $\mathrm{Z}$ boson. In the case of charged leptons the corrections turn out to be at the level of $-3 \%$ for inclusive cross sections. For the decay into neutrinos the corrections are negligible, when standard cuts are applied, but grow to $-10 \%$ for cuts inspired by ATLAS searches for new physics [1]. In the high-energy tails of distributions the corrections are in all channels of several tens of percent.

12th International Symposium on Radiative Corrections (Radcor 2015) and LoopFest XIV (Radiative Corrections for the LHC and Future Colliders)

15-19 June, 2015

UCLA Department of Physics \& Astronomy Los Angeles, USA

\footnotetext{
* Speaker.
} 


\section{Introduction}

The main task of present and future LHC physics is the precise study of the Standard Model (SM) and the search for new physics. Theoretical phenomenology must therefore be able to produce reliable predictions for SM processes with high particle multiplicity, and NLO accuracy must be reached for collisions involving many particles in the final state. Beyond QCD corrections, which are usually dominant at hadron colliders, we should consider electroweak (EW) corrections as well, since they can have an important impact, e.g. in differential distributions at high energies. Many codes are available for the computation of elementary processes in QCD at NLO $[2,3,4,5,6,7,8]$ and a big effort is made to cover also EW corrections $[9,10,11]$. Here we present the features of the code RECOLA [9], which computes NLO amplitudes in the full SM. The automatization is achieved choosing an alternative approach to Feynman diagrams, based on recursion relations for off-shell currents. We have used RECOLA to calculate EW corrections to the processes $\mathrm{pp} \rightarrow l^{+} l^{-}+2$ jets and $\mathrm{pp} \rightarrow v \bar{v}+2$ jets, whose results are also presented.

\section{Structure of RECOLA}

The code is written in FORTRAN90 and can currently only be used within fortran modules or fortran programs. Every fortran module calling RECOLA has to include the module recola through the statement use recola. The call of RECOLA is then achieved in three basic steps:

- First the user defines the processes to be computed:

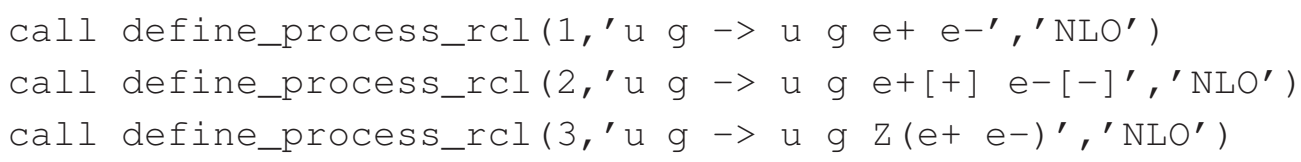

The integer argument is the process id, while the last argument specifies the loop order (LO or NLO). The process is given through a string, where incoming and outgoing particles are separated by the sybmbol ->; specific helicity contributions are selected by the symbols $[+],[-],[0]$, while the round brackets are used to specify intermediate productions and decays.

- The skeleton of the recursion procedure is then generated for all defined processes:

call generate_processes_rcl

- Finally the amplitudes are computed calling the subroutine compute_process_rcl with the process id of the defined processes:

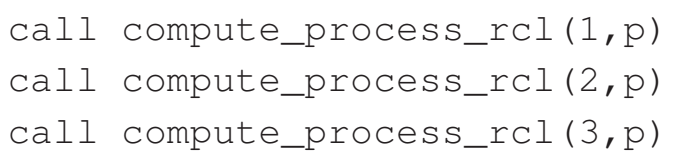

The first two steps have to be run just once, while compute_process_rcl needs to be called for each phase-space point with momentum $\mathrm{p}(0: 3,1:$ legs $)$.

Here we give an example of a simple program calling RECOLA for the computation of one phasespace point for the process $\mathrm{u} \overline{\mathrm{u}} \rightarrow \mathrm{gge}^{+} \mathrm{e}^{-}$: 


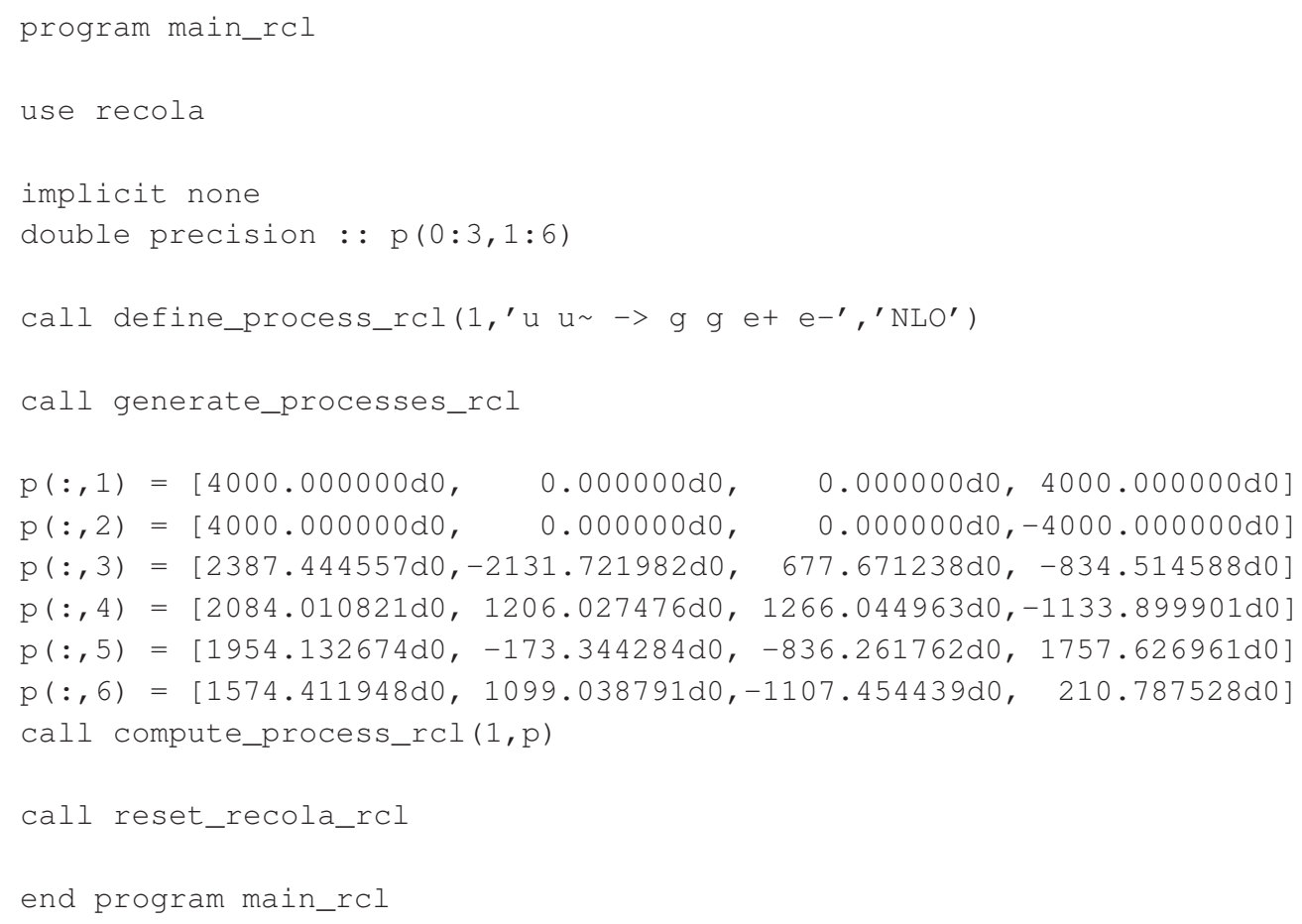

\section{Features of RECOLA}

RECOLA has been designed for the computation of Born and one-loop amplitudes in the full SM. Tree-level amplitudes are calculated using Dyson-Schwinger equations [12], while the recursion relations for the one-loop amplitudes are based on the decomposition of one-loop amplitudes in terms of tensor integrals (TIs) $T_{(t)}^{\mu_{1} \cdots \mu_{r_{t}}}$ and tensor coefficients (TCs) $c_{\mu_{1} \cdots \mu_{r_{t}}}^{(t)}$. The TCs are computed recursively by RECOLA, while the computation of the TIs relies on the external library COLLIER [13]. The indices $\mu_{1}, \ldots, \mu_{r_{t}}$ are strictly 4-dimensional and the correct amplitude is obtained by adding a rational part of type R2 [14]. Renormalization is performed making use of on-shell counterterms for the EW sector and an $\overline{\mathrm{MS}}$ counterterm for the strong coupling constant (ultraviolet finiteness can be checked numerically). The user can fix different values of the strong coupling constant at each phase-space point through the dynamical running of $\alpha_{\mathrm{s}}$. Unstable particles are consistently implemented by introducing an imaginary part for the squared masses in the framework of the complex-mass scheme [15]. For each given process the user has the possibility to select/unselect arbitrary powers in $\alpha_{\mathrm{s}}$. Intermediate production and decays can be also selected and a consistent computation of the factorisable corrections of the pole approximation is implemented. Soft and collinear divergences can be treated either in dimensional or mass regularization. Various optimizations have been implemented in the treatment of colour and in the sum over helicity configurations, in order to maximally exploit the properties of recursion relations for off-shell currents. In addition to LO and NLO amplitudes and squared amplitudes, RECOLA calculates all LO colourand spin-correlated amplitudes needed for the application of the dipole subtraction method [16].

\section{Performances of RECOLA}

The amount of memory for executables, object files and libraries is usually negligible, while 
the RAM needed does not exceed 2 Gbyte even for complicated processes. In order to give an idea of the efficiency of RECOLA we present the CPU time needed for the computation of some processes of physical interest at the LHC. The generation time is given by $t_{\mathrm{gen}}$, while $t_{\mathrm{TIs}}$ and $t_{\mathrm{TCs}}$ give the CPU time per phase-space point for the computation of the tensor integrals (with COLLIER) and the tensor coefficients respectively ${ }^{1}$ :

- $\mathrm{QCD}$ corrections $\left(\mathrm{W}^{+} \rightarrow \mathrm{e}^{+} v_{e}, \mathrm{~W}^{-} \rightarrow \mathrm{e}^{-} \bar{v}_{e}\right.$, colour and helicity summed):

$$
\begin{array}{lrrrrrr}
\mathrm{u} \overline{\mathrm{d}} \rightarrow \mathrm{W}^{+} \mathrm{gg} & t_{\mathrm{gen}}: & 2.4 \mathrm{~s} & t_{\mathrm{TIs}}: & 4.0 \mathrm{~ms} & t_{\mathrm{TCS}}: & 1.1 \mathrm{~ms} \\
\mathrm{u} \overline{\mathrm{d}} \rightarrow \mathrm{W}^{+} \mathrm{ggg} & t_{\mathrm{gen}}: & 15 \mathrm{~s} & t_{\mathrm{TIs}}: & 67 \mathrm{~ms} & t_{\mathrm{TCs}}: & 45 \mathrm{~ms} \\
\mathrm{u} \overline{\mathrm{u}} \rightarrow \mathrm{W}^{+} \mathrm{W}^{-} \mathrm{gg} & t_{\mathrm{gen}}: & 76 \mathrm{~s} & t_{\mathrm{TIs}}: & 83 \mathrm{~ms} & t_{\mathrm{TCs}}: & 16 \mathrm{~ms}
\end{array}
$$

\begin{tabular}{|c|c|c|c|c|c|c|}
\hline $\mathrm{u} \overline{\mathrm{u}} \rightarrow \mathrm{e}^{+} \mathrm{e}^{-} \mathrm{gg}$ & $t_{\mathrm{gen}}:$ & $3.2 \mathrm{~s}$ & $t_{\mathrm{TIs}}:$ & $27 \mathrm{~ms}$ & $t_{\mathrm{TCs}}:$ & $25 \mathrm{~ms}$ \\
\hline $\mathrm{e}^{-} \mathrm{u} \overline{\mathrm{u}}$ & $t_{\mathrm{gen}}:$ & $5 \mathrm{~s}$ & $t_{\mathrm{TIs}}:$ & $68 \mathrm{~ms}$ & $t_{\mathrm{TCs}}:$ & ns \\
\hline $\operatorname{ggg}$ & $t_{\mathrm{gen}}:$ & $44 \mathrm{~s}$ & $t_{\mathrm{TIs}}:$ & $331 \mathrm{~ms}$ & $t_{\mathrm{TCs}}:$ & $4 \mathrm{~ms}$ \\
\hline $\mathrm{i} \rightarrow \mathrm{e}^{+} \mathrm{e}^{-} \mathrm{u} \overline{\mathrm{u}} \mathrm{g}$ & gen: & $50 \mathrm{~s}$ & $t_{\mathrm{TIs}}:$ & $835 \mathrm{~ms}$ & $t_{\mathrm{TCs}}:$ & 632 \\
\hline
\end{tabular}

- $\mathrm{EW}+\mathrm{QCD}$ corrections (colour and helicity summed):

\section{Production of lepton-antilepton with two jets at the LHC}

We have studied with RECOLA the production of a $\mathrm{Z}$ boson accompanied by two jets. This process is a background to Higgs-boson production in vector-boson fusion [18, 19], particularly important when the two jets are in forward and backward rapidity regions. In addition, if the $Z$ boson decays into neutrinos, the process $\mathrm{pp} \rightarrow Z+2$ jets has the same signature (missing energy plus 2 jets) as the production of a pair of squark and anti-squark, each subsequently decaying into a jet and an invisible neutralino. Such events are mainly searched for in high-energy regions, where EW corrections are usually sizable. Moreover, the experimental estimation of the irreducible SM background is usually obtained by data-driven extrapolations, based on the SM process pp $\rightarrow$ $Z+2$ jets $\rightarrow l^{+} l^{-}+2$ jets.

Analysing in full generality the process $\mathrm{pp} \rightarrow l \bar{l}+2$ jets (where $l$ can be either a charged lepton or a neutrino and $\bar{l}$ is its antiparticle), we notice that the LO amplitude gets contributions from QCD (gluonic or four-quark) diagrams and from EW (four-quark) ones (see Fig. 1).
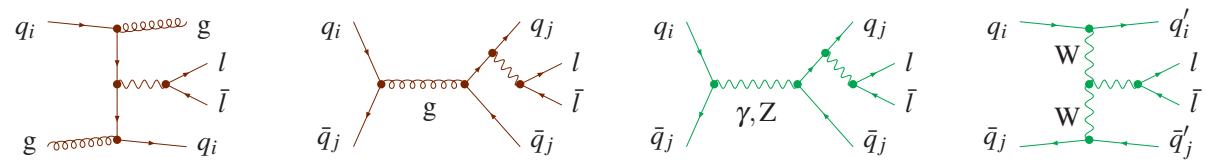

Figure 1: Sample tree diagrams of QCD gluonic contributions (first diagram), QCD four-quark contributions (second diagram) and EW four-quark contributions (third and fourth diagram).

The NLO cross section has been only partially studied up to now: the $\mathscr{O}\left(\alpha^{2} \alpha_{\mathrm{s}}^{3}\right)$ corrections have been computed in [20], the QCD corrections to EW LO contributions with vector-bosonfusion topology (a gauge-invariant subset of the $\mathscr{O}\left(\alpha^{4} \alpha_{\mathrm{s}}\right)$ corrections) have been investigated in [19] and the effects of EW Sudakov logarithms have been studied in NLL approximation in [21] for $\mathrm{pp} \rightarrow \mathrm{jj} v \bar{v}$. Very recently, the complete QCD-EW NLO corrections have been computed in [22]. Here we present our computation of the $\mathscr{O}\left(\alpha^{3} \alpha_{\mathrm{s}}^{2}\right)$ corrections for pp $\rightarrow l^{+} l^{-}+2$ jets $[9,23]$ and our preliminary results for $\mathrm{pp} \rightarrow v \bar{v}+2$ jets [24].

\footnotetext{
${ }^{1}$ The numbers have been produced averaging the CPU time of 100 phase-space points generated by RAMBO [17] on a personal computer with processor Intel(R) Core(TM) i5-2450M CPU @ 2.50 GHz.
} 


\begin{tabular}{|c|c|c|c|c|}
\hline Process class & $\sigma^{\mathrm{LO}}[\mathrm{pb}]$ & $\sigma^{\mathrm{LO}} / \sigma_{\mathrm{tot}}^{\mathrm{LO}}[\%]$ & $\sigma_{\mathrm{EW}}^{\mathrm{NLO}}[\mathrm{pb}]$ & $\frac{\sigma_{\mathrm{EW}}^{\mathrm{NLO}}}{\sigma^{\mathrm{LO}}}-1[\%]$ \\
\hline \multicolumn{5}{|l|}{ Basic cuts } \\
\hline gluonic & $40.910(8)$ & 79.9 & $39.932(9)$ & -2.39 \\
\hline four-quark & $10.299(1)$ & 20.1 & $10.033(1)$ & -2.58 \\
\hline sum & $51.209(8)$ & 100 & $49.965(9)$ & -2.43 \\
\hline bottom quarks & $4.376(3)$ & 8.54 & & \\
\hline \multicolumn{5}{|l|}{ VBF cuts } \\
\hline gluonic & $0.6178(4)$ & 59.4 & $0.5992(3)$ & -3.01 \\
\hline four-quark & $0.4217(1)$ & 40.6 & $0.4102(1)$ & -2.73 \\
\hline sum & $1.0396(4)$ & 100 & $1.0093(3)$ & -2.91 \\
\hline bottom quarks & $0.05182(2)$ & 4.98 & & \\
\hline
\end{tabular}

Table 1: Composition of the LO and NLO total cross section for $\mathrm{pp} \rightarrow l^{+} l^{-}+2$ jets at the LHC operating at $13 \mathrm{TeV}$ for basic and VBF cuts.

\subsection{General setup}

For the calculation we work in the $G_{\mu}$ scheme, i.e. we fix the value of $\alpha$ via its tree-level relation with the Fermi constant $G_{\mu}$ :

$$
\alpha_{G_{\mu}}=\frac{\sqrt{2} G_{\mu} M_{\mathrm{W}}^{2}}{\pi}\left(1-\frac{M_{\mathrm{W}}^{2}}{M_{\mathrm{Z}}^{2}}\right) .
$$

In order to appropriately treat potentially resonant $\mathrm{Z}$ and $\mathrm{W}$ propagators, we consistently use the complex-mass scheme [15]. The virtual amplitudes are calculated in the 't Hooft-Feynman gauge, and all light fermions are considered massless. We employ the MSTW2008LO PDF set [25] and choose $\mu_{R}=\mu_{F}=M_{\mathrm{Z}}$ for the QCD factorization and renormalization scales. Jets are constructed with the anti- $k_{\mathrm{T}}$ algorithm [26] with separation parameter $\mathrm{R}=0.4$. Using the same clustering algorithm with $\mathrm{R}=0.4$, photons and charged leptons are recombined into leptons, and quarks/gluons and photons are recombined into jets. In order to have IR-safe observables, we discard photonic jets consisting of a parton $a\left(a=q_{i}, \bar{q}_{i}, \mathrm{~g}\right)$ and a hard photon with $z_{\gamma}=E_{\gamma} /\left(E_{\gamma}+E_{a}\right)$ above a critical value $z_{\gamma}^{\text {cut }}=0.7$ and absorb the left-over collinear singularities into the quark-photon fragmentation function [27].

5.2 Results for the process $\mathrm{pp} \rightarrow l^{+} l^{-}+2$ jets

In order to select a final state of well separated hard lepton, hard antilepton and hard jets, we impose the followig cuts:

$$
p_{\mathrm{T}, \mathrm{j}}>30 \mathrm{GeV}, \quad\left|y_{\mathrm{j}}\right|<4.5, \quad p_{\mathrm{T}, l}>20 \mathrm{GeV}, \quad\left|y_{l}\right|<2.5, \quad \Delta R_{l^{-} l^{+}}>0.2, \quad \Delta R_{\mathrm{lj}}>0.5 .
$$

We study the process in two configurations requiring following additional cuts:

- Basic cuts: $66 \mathrm{GeV}<M_{l^{-} l^{+}}<116 \mathrm{GeV}$;

- VBF cuts: $M_{\mathrm{jj}}>600 \mathrm{GeV}, \quad\left|y_{\mathrm{j}_{1}}-y_{\mathrm{j}_{2}}\right|>4, \quad y_{\mathrm{j}_{1}} y_{\mathrm{j}_{2}}<0, \min \left(y_{\mathrm{j}_{1}}, y_{\mathrm{j}_{2}}\right)<y_{l}<\max \left(y_{\mathrm{j}_{1}}, y_{\mathrm{j}_{2}}\right)$.

In Table 1 the results for the total cross section can be found. We note that parton interactions involving external gluons dominate the total cross section (also with the VBF cuts), while partonic processes with external bottom quarks do not contribute much to the total cross section. The 

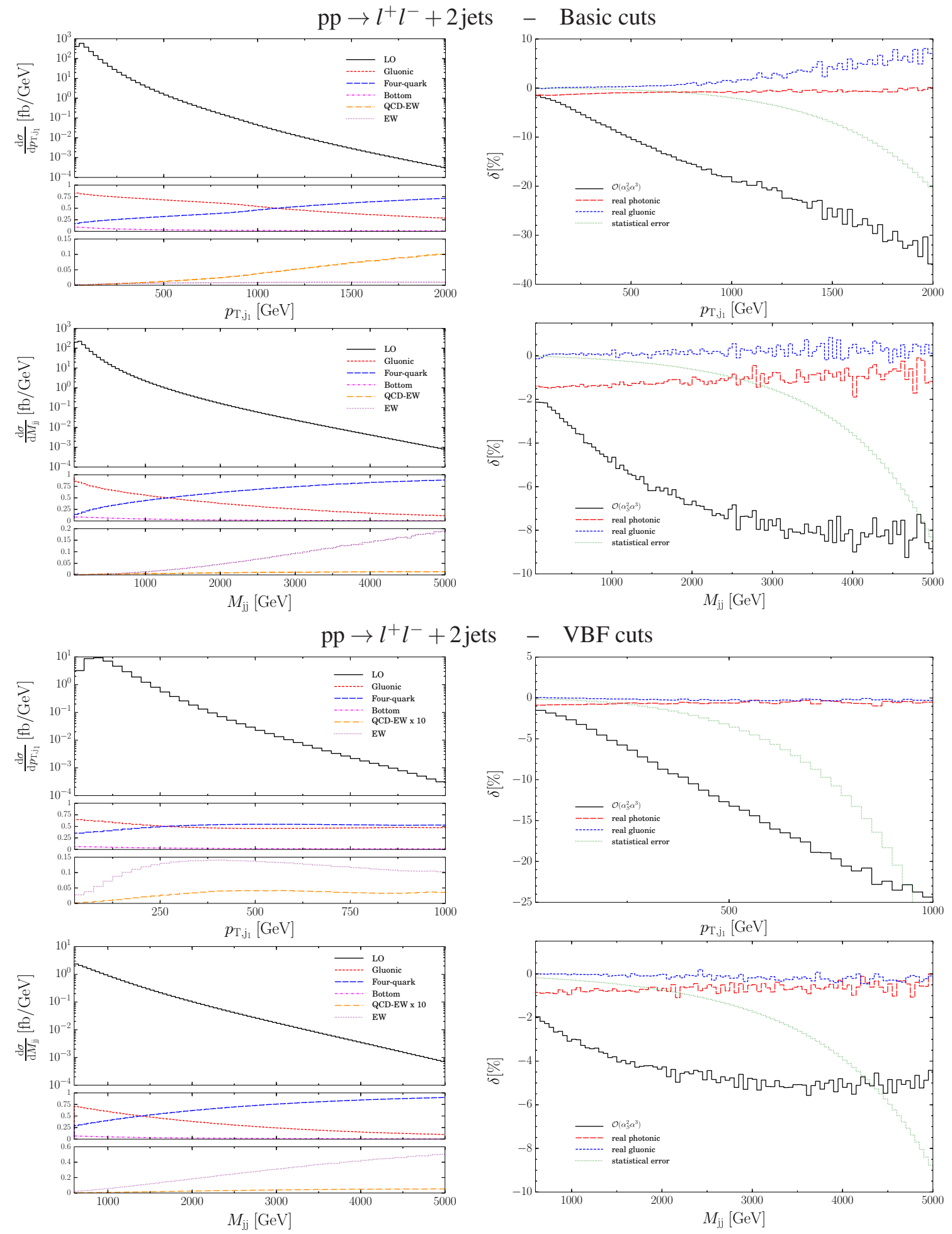

Figure 2: Distributions of the transverse-momentum of the hardest jet and for the di-jet invariant-mass for $\mathrm{pp} \rightarrow l^{+} l^{-}+2$ jets at the $13 \mathrm{TeV}$ LHC. Left: the total LO distributions (black), the relative gluonic (red), four-quark (blue) and bottom (magenta) contributions, the relative contributions of the squared EW diagrams (purple) and QCD-EW interference (orange). Right: the relative NLO corrections (black), the included real photonic (red) and real gluonic (blue) corrections, the statistical error (green) for an integrated luminosity of $300 \mathrm{fb}^{-1}$. 


\begin{tabular}{|c|c|c|c|c|}
\hline Process class & $\sigma^{\mathrm{LO}}[\mathrm{pb}]$ & $\sigma^{\mathrm{LO}} / \sigma_{\mathrm{tot}}^{\mathrm{LO}}[\%]$ & $\sigma_{\mathrm{EW}}^{\mathrm{NLO}}[\mathrm{pb}]$ & $\frac{\sigma_{\mathrm{EW}}^{\mathrm{NLO}}}{\sigma^{\mathrm{LO}}}-1[\%]$ \\
\hline \multicolumn{5}{|l|}{ Basic cuts } \\
\hline gluonic & $456.94(11)$ & 81.5 & $454.34(11)$ & -0.57 \\
\hline four-quark & $103.66(01)$ & 18.5 & $102.40(02)$ & -1.22 \\
\hline sum & $560.60(11)$ & 100 & $556.74(11)$ & -0.69 \\
\hline bottom quarks & $37.699(6)$ & 6.72 & & \\
\hline \multicolumn{5}{|l|}{ ATLAS cuts } \\
\hline gluonic & $1.64947(39)$ & 72.7 & $1.47947(42)$ & -10.31 \\
\hline four-quark & $0.61850(07)$ & 27.3 & $0.55783(16)$ & -9.81 \\
\hline sum & $2.26797(39)$ & 100 & $2.03729(45)$ & -10.17 \\
\hline bottom quarks & 0.07969(01) & 3.51 & & \\
\hline
\end{tabular}

Table 2: Composition of the LO and NLO total cross section for $\mathrm{pp} \rightarrow v \bar{v}+2$ jets at the LHC operating at $13 \mathrm{TeV}$ for basic and ATLAS cuts.

$\mathscr{O}\left(\alpha^{3} \alpha_{\mathrm{s}}^{2}\right)$ corrections to the total cross section of both gluonic and four-quark channels are small and negative for both sets of cuts.

In Fig. 2 we present the differential distributions for the transverse-momentum of the hardest jet $p_{\mathrm{T}, \mathrm{j}_{1}}$ (jet with highest transverse-momentum) and for the di-jet invariant-mass $M_{\mathrm{jj}}$. Looking at the LO distributions we notice that the cross section drops by several orders of magnitude in the considered acceptance cuts, when high values of $p_{\mathrm{T}, \mathrm{j}_{1}}$ and $M_{\mathrm{jj}}$ are reached. When basic cuts are applied, gluonic processes dominate for low $p_{\mathrm{T}, \mathrm{j}_{1}}$ and low $M_{\mathrm{jj}}$, while for high values the four-quark processes become more important. The same happens for the invariant-mass distribution with the VBF cuts, while for the transverse-momentum distribution the four-quark and gluonic contributions are similar above $270 \mathrm{GeV}$. It is important to notice that at high values of the invariant-mass EW LO diagrams contribute considerably (up to $50 \%$ for VBF cuts). On the right-hand side of the figure we see that one-loop corrections exhibit the typical enhancement at high energies, where sizable logarithms of EW origin appear. This effect is much larger for the transverse-momentum distribution (in particular for the VBF cuts) than for the invariant-mass distribution. This is partially due to the fact that for high invariant-mass EW LO diagrams contribute considerably and, since we do not include the corresponding EW corrections of order $\mathscr{O}\left(\alpha^{5}\right)$, we miss part of the contributions with large EW logarithms. The drop of the cross section for high energies causes an increase of the statistical error, which however is always smaller than the corrections in the considered range. In general the impact of real-subtracted photon emission is small, while, when basic cuts are applied, the real-subtracted gluon emission increases for high $p_{\mathrm{T}, j_{1}}$ values up to $8 \%$.

\subsection{Results for the process $\mathrm{pp} \rightarrow v \bar{v}+2$ jets}

We study the process imposing two sets of acceptance cuts:

- Basic cuts: $p_{\mathrm{T}, \mathrm{j}}>30 \mathrm{GeV}, \quad\left|y_{j}\right|<4.5, \quad E_{\mathrm{T}}>25 \mathrm{GeV}$;

- ATLAS cuts: $p_{\mathrm{T}, \mathrm{j}_{1}}>130 \mathrm{GeV}, \quad p_{\mathrm{T}, \mathrm{j}_{2}}>60 \mathrm{GeV}, \quad\left|y_{j}\right|<4.5$,

$$
H_{\mathrm{T}}>800 \mathrm{GeV}, \quad E_{\mathrm{T}}>8 \sqrt{H_{\mathrm{T}}} \sqrt{\mathrm{GeV}}, \quad \Delta \phi_{E_{\mathrm{T} j}}>0.4 .
$$

These cuts are inspired by ATLAS searches for gluino and/or squark production involving final states with large missing transverse-momentum [1]. 

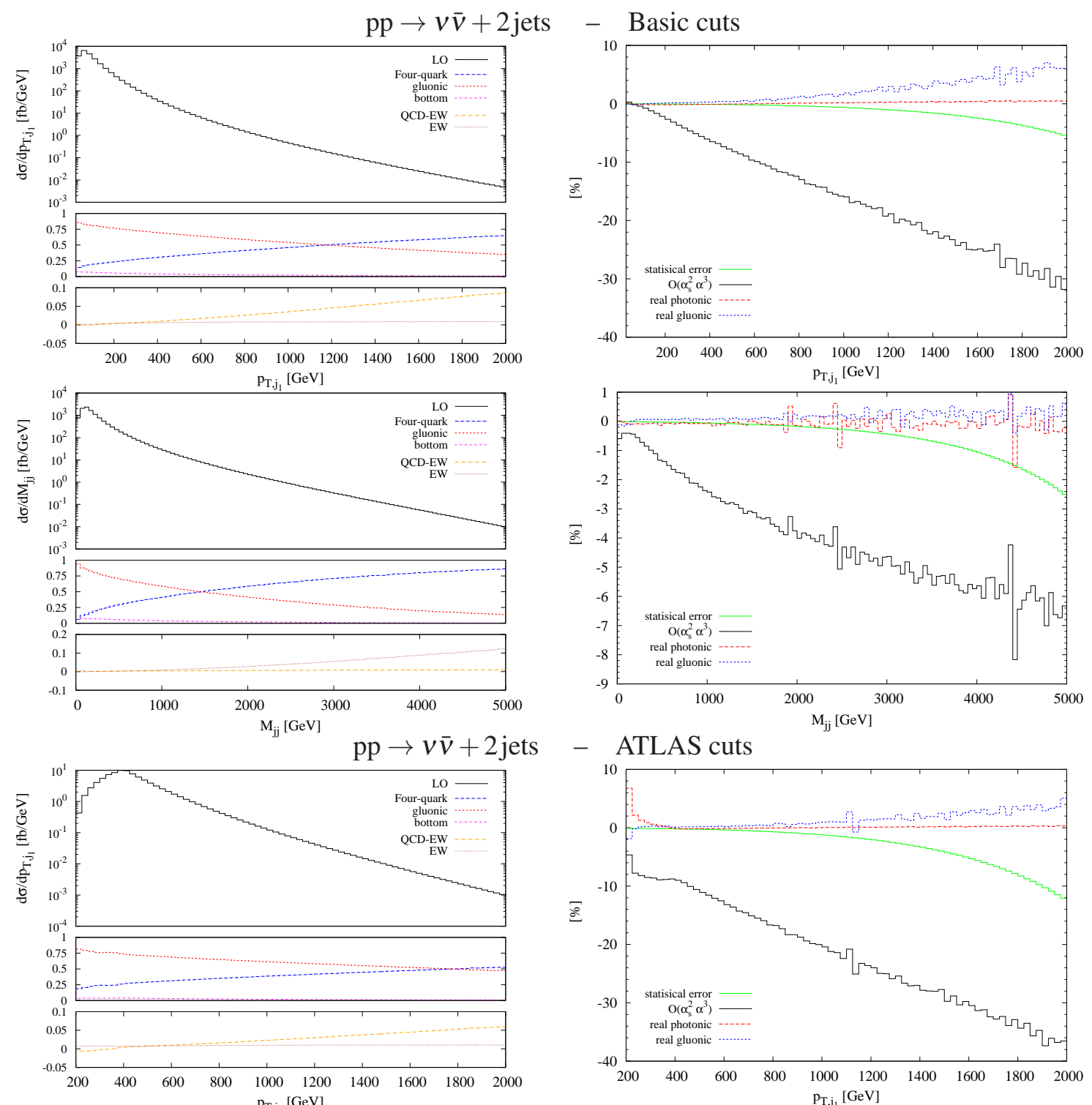

- ATLAS cuts
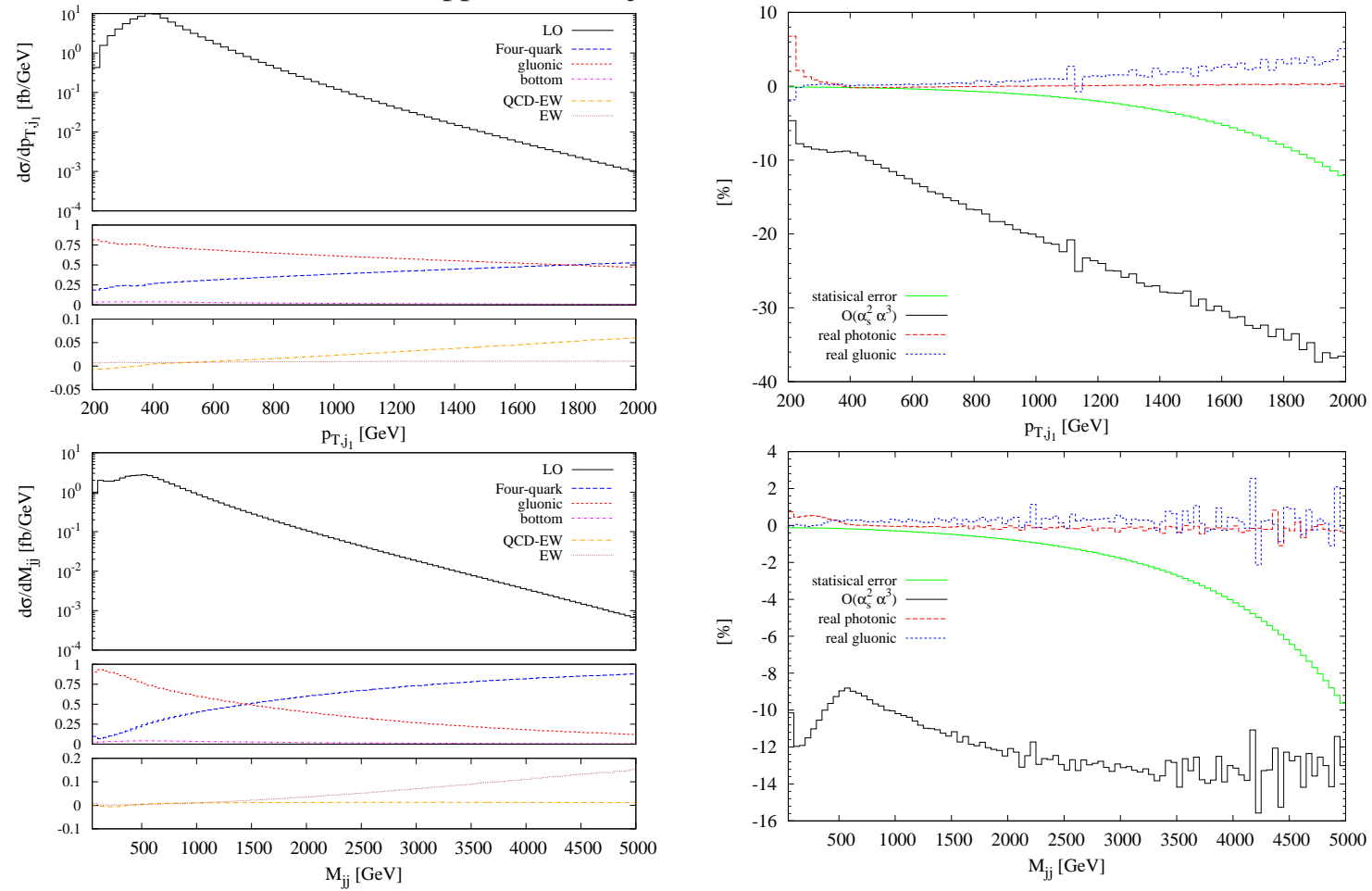

Figure 3: Distributions of the transverse-momentum of the hardest jet and for the di-jet invariant-mass for $\mathrm{pp} \rightarrow v \bar{v}+2$ jets at the $13 \mathrm{TeV}$ LHC. Left: the total LO distributions (black), the relative gluonic (red), fourquark (blue) and bottom (magenta) contributions, the relative contributions of the squared EW diagrams (purple) and QCD-EW interference (orange). Right: the relative NLO corrections (black), the included real photonic (red) and real gluonic (blue) corrections, the statistical error (green) for an integrated luminosity of $300 \mathrm{fb}^{-1}$. 
In Table 2 we present the preliminary results for the total cross section. Comparing the results for basic cuts with those of $\mathrm{pp} \rightarrow l^{+} l^{-}+2$ jets (for a single lepton flavour), the production rate is more than a factor of ten larger, mainly because of the higher branching ratios of $\Gamma_{\mathrm{Z} \rightarrow v v} / \Gamma_{\mathrm{Z}}^{\text {tot }}$ with respect to $\Gamma_{\mathrm{Z} \rightarrow l^{+} l^{-}} / \Gamma_{\mathrm{Z}}^{\text {tot }}$. The relative importance of the gluonic and four-quark channels is similar to the case of $\mathrm{pp} \rightarrow l^{+} l^{-}+2$ jets. With the ATLAS cuts the cross section is reduced by a factor 250 with respect to basic cuts and the gluonic channels still dominate. The $\mathscr{O}\left(\alpha^{3} \alpha_{\mathrm{s}}^{2}\right)$ corrections to the total cross section of gluonic and four-quark channels are again negative for both cuts. The size of the corrections is negligible when basic cuts are applied, but is around $-10 \%$ for ATLAS cuts.

In Fig. 3 we present the differential distributions for the transverse-momentum of the hardest jet $p_{\mathrm{T}, \mathrm{j}_{1}}$ (jet with highest transverse-momentum) and for the di-jet invariant-mass $M_{\mathrm{jj}}$. As happened for charged lepton production, the cross section drops by several orders of magnitude in the considered acceptance cuts for high values of $p_{\mathrm{T}, \mathrm{j}_{1}}$ and $M_{\mathrm{jj}}$. When basic cuts are applied the LO plots only differ from those for $\mathrm{pp} \rightarrow l^{+} l^{-}+2$ jets by an overall factor 10 in the magnitude. For ATLAS cuts we notice that the distributions increase in the low $p_{\mathrm{T}, \mathrm{j}_{1}}$ and $M_{\mathrm{jj}}$ regions, mainly because of the cut imposed on the missing transverse-momentum. While for low $p_{\mathrm{T}, \mathrm{j}_{1}}$ and $M_{\mathrm{jj}}$ the cross section is three orders of magnitude smaller than with basic cuts, in the tail of the distribution the difference is less than a factor of ten. The four-quark contributions equal the gluonic ones for $p_{\mathrm{T}, \mathrm{j}_{1}}>1.5 \mathrm{TeV}$ when basic cuts are applied. We also notice that the importance of the EW LO contributions in the tail of the invariant-mass distribution is less enhanced than for $\mathrm{pp} \rightarrow l^{+} l^{-}+2$ jets (for both cuts). One-loop corrections are negative and present the same enhancement of EW origin at high energies as for charged lepton production. The smaller statistical error is due to the larger cross section in the case of neutrino production. For basic cuts the real gluon emission increases at high $p_{\mathrm{T}, \mathrm{j}_{1}}$ (as for $\mathrm{pp} \rightarrow l^{+} l^{-}+2 \mathrm{jets}$ ), while the real photon emission in the invariant-mass destribution is smaller than for $\mathrm{pp} \rightarrow l^{+} l^{-}+2$ jets, because of the neutrino in the final state. When ATLAS cuts are applied we notice that the corrections are of $10 \%$ at low $p_{\mathrm{T}, \mathrm{j}_{1}}$ and $M_{\mathrm{jj}}$; they increase for high transverse-momentum, while remaining almost constant in the invariant-mass.

\section{Conclusion}

We have presented the properties and discussed the performance of the code RECOLA for the computation of EW and QCD elementary processes in the SM at NLO, based on a one-loop generalization of Dyson-Schwinger recursion relations. The analysis of EW corrections to the process $\mathrm{pp} \rightarrow l^{+} l^{-}+2$ jets and preliminary results for the process $\mathrm{pp} \rightarrow v \bar{v}+2$ jets have also been shown.

\section{Acknowledgements}

This work was supported in part by the Deutsche Forschungsgemeinschaft (DFG) under reference number DE 623/2-1. The work of L.H. was supported by the grants FPA2013-46570-C2-1-P and 2014-SGR-104, and partially by the Spanish MINECO under the project MDM-2014-0369 of ICCUB (Unidad de Excelencia 'María de Maeztu').

\section{References}

[1] G. Aad et al. [ATLAS Collaboration], JHEP 1409 (2014) 176 [arXiv:1405.7875 [hep-ex]]. 
[2] K. Arnold et al., Comput. Phys. Commun. 180 (2009) 1661 [arXiv:0811.4559 [hep-ph]].

[3] C. F. Berger et al., Phys. Rev. D 78 (2008) 036003 [arXiv:0803.4180 [hep-ph]].

[4] S. Badger, B. Biedermann and P. Uwer, Comput. Phys. Commun. 182 (2011) 1674 [arXiv:1011.2900 [hep-ph]].

[5] V. Hirschi et al., JHEP 1105 (2011) 044 [arXiv:1103.0621 [hep-ph]].

[6] G. Bevilacqua et al., Comput. Phys. Commun. 184 (2013) 986 [arXiv:1110.1499 [hep-ph]].

[7] G. Cullen et al., Eur. Phys. J. C 72 (2012) 1889 [arXiv:1111.2034 [hep-ph]].

[8] F. Cascioli, P. Maierhofer and S. Pozzorini, Phys. Rev. Lett. 108 (2012) 111601 [arXiv:1111.5206 [hep-ph]].

[9] S. Actis et al., JHEP 1304 (2013) 037 [arXiv:1211.6316 [hep-ph]]; PoS RADCOR 2013 (2013) 034 [arXiv:1311.6662 [hep-ph]]; PoS LL 2014 (2014) 023.

[10] S. Kallweit et al., JHEP 1504 (2015) 012 [arXiv:1412.5157 [hep-ph]]; arXiv:1505.05704 [hep-ph].

[11] S. Frixione et al., JHEP 1409 (2014) 065 [arXiv:1407.0823 [hep-ph]]; JHEP 1506 (2015) 184 [arXiv:1504.03446 [hep-ph]].

[12] F. J. Dyson, Phys. Rev. 75 (1949) 1736; J. S. Schwinger, Proc. Nat. Acad. Sci. 37 (1951) 452; Proc. Nat. Acad. Sci. 37 (1951) 455.

[13] A. Denner and S. Dittmaier, Nucl. Phys. B 658 (2003) 175 [hep-ph/0212259]; Nucl. Phys. B 734 (2006) 62 [hep-ph/0509141]; Nucl. Phys. B 844 (2011) 199 [arXiv:1005.2076 [hep-ph]]; A. Denner, S. Dittmaier and L. Hofer, PoS LL 2014 (2014) 071 [arXiv:1407.0087 [hep-ph]].

[14] G. Ossola, C. G. Papadopoulos and R. Pittau, JHEP 0805 (2008) 004 [arXiv:0802.1876 [hep-ph]]; P. Draggiotis, M. V. Garzelli, C. G. Papadopoulos and R. Pittau, JHEP 0904 (2009) 072 [arXiv:0903.0356 [hep-ph]]; M. V. Garzelli, I. Malamos and R. Pittau, JHEP 1001 (2010) 040 [Erratum-ibid. 1010 (2010) 097] [arXiv:0910.3130 [hep-ph]].

[15] A. Denner, S. Dittmaier, M. Roth and D. Wackeroth, Nucl. Phys. B 560 (1999) 33 [hep-ph/9904472]; A. Denner, S. Dittmaier, M. Roth and L. H. Wieders, Nucl. Phys. B 724 (2005) 247 [Erratum-ibid. B 854 (2012) 504] [hep-ph/0505042]; A. Denner and S. Dittmaier, Nucl. Phys. Proc. Suppl. 160 (2006) 22 [hep-ph/0605312]; A. Denner and J. N. Lang, Eur. Phys. J. C 75 (2015) 8, 377 [arXiv:1406.6280 [hep-ph]].

[16] S. Catani and M. H. Seymour, Nucl. Phys. B 485, 291 (1997) [Erratum-ibid. B 510, 503 (1998)].

[17] R. Kleiss, W. J. Stirling and S. D. Ellis, Comput. Phys. Commun. 40 (1986) 359.

[18] V. A. Khoze, M. G. Ryskin, W. J. Stirling and P. H. Williams, Eur. Phys. J. C 26 (2003) 429 [hep-ph/0207365].

[19] C. Oleari and D. Zeppenfeld, Phys. Rev. D 69 (2004) 093004 [hep-ph/0310156].

[20] J. M. Campbell and R. K. Ellis, Phys. Rev. D 65 (2002) 113007 [hep-ph/0202176]; J. M. Campbell, R. K. Ellis and D. L. Rainwater, Phys. Rev. D 68 (2003) 094021 [hep-ph/0308195].

[21] M. Chiesa, et al., Phys. Rev. Lett. 111 (2013) 12, 121801 [arXiv:1305.6837 [hep-ph]].

[22] S. Kallweit et al., arXiv:1511.08692 [hep-ph].

[23] A. Denner, L. Hofer, A. Scharf and S. Uccirati, PoS RADCOR 2013 (2013) 019 [arXiv:1311.5336 [hep-ph]]; PoS LL 2014 (2014) 058; JHEP 1501, 094 (2015) [arXiv:1411.0916 [hep-ph]].

[24] A. Denner, L. Hofer, A. Scharf and S. Uccirati, in preparation.

[25] A. D. Martin et al., Eur. Phys. J. C 63, (2009) 189 [arXiv:0901.0002 [hep-ph]].

[26] M. Cacciari, G. P. Salam and G. Soyez, JHEP 0804 (2008) 063 [arXiv:0802.1189 [hep-ph]].

[27] E. W. N. Glover and A. G. Morgan, Z. Phys. C 62 (1994) 311; D. Buskulic et al. [ALEPH Collaboration], Z. Phys. C 69 (1996) 365. 